\title{
First NACO observations of the Brown Dwarf LHS 2397aB ${ }^{\star}$
}

\author{
E. Masciadri ${ }^{1}$, W. Brandner ${ }^{1}$, H. Bouy ${ }^{2}$, R. Lenzen ${ }^{1}$, A. M. Lagrange ${ }^{3}$, and F. Lacombe ${ }^{4}$ \\ 1 Max-Plank Institut für Astronomie, Königstuhl 17, 69117 Heidelberg, Germany \\ 2 European Southern Observatory, 85748 Garching bei München, Germany \\ ${ }^{3}$ Laboratoire d'Astrophysique, Observatoire de Grenoble, 414 rue de la Piscine, BP 53, 38041 Grenoble Cedex 9, France \\ ${ }^{4}$ Observatoire Paris-Meudon, LESIA, place Jules Janssen, 92195 Meudon Cedex, France
}

Received 27 March 2003/ Accepted 19 August 2003

\begin{abstract}
Observations of the standard late type M8 star LHS 2397aA were obtained at the ESO-VLT 8 m telescope "Yepun" using the NAOS/CONICA Adaptive Optics facility. The observations were taken during the NACO commissioning, and the infrared standard star LHS 2397aA was observed in the $H$, and $K$ s broad band filters. In both bands the brown dwarf companion LHS 2397aB was detected. Using a program recently developed (Bouy et al. 2003) for the detection of stellar binaries we calculated the principal astrometric parameters (angular binary separation and position angle PA) and the photometry of LHS 2397aA and LHS 2397aB. Our study largely confirms previous results obtained with the AO-Hokupa'a facility at Gemini-North (Freed et al. 2003); however a few discrepancies are observed.
\end{abstract}

Key words. stars: low-mass, brown dwarfs - stars: binaries: general - techniques: high angular resolution

\section{Introduction}

The study of the frequency of brown dwarf (BD) companions at different distances from the primary is a crucial point for the determination of the origin of these sub-stellar objects $\left(\sim[13-75] M_{J}\right)$. The most popular scenarios are: (1) the BDs are the result of a cloud fragmentation mechanism (Elmegreen 1999). After the formation of a nucleus, the BDs would be built by accretion from a disc like star, (2) the BDs are the result of a disk fragmentation mechanism (Pickett et al. 2000), (3) the BDs are ejected stellar embryos (Reipurth \& Clarke 2001). In the last years several BDs companions of Sun-like stars (Marcy et al. 2003; Gizis et al. 2001; Kirkpatrick et al. 2001; Potter et al. 2002) and of very low mass (VLM) stars and/or other BDs (Mártin et al. 2000; Koerner et al. 1999; Bouy et al. 2003; Close et al. 2003; Burgasser et al. 2003) were discovered. These discoveries can provide useful information for a statistical analysis of the BD binary characteristics (orbital parameters, mass ratio, and so on) and BD binary frequency as a function of the separation. We refer the reader to the papers by Bouy et al. (2003), Close et al. (2003) and Burgasser et al. (2003) for more complete discussions of the subject using the most recent statistical results.

\footnotetext{
Send offprint requests to: E. Masciadri, e-mail: masciadri@mpia.de

* Based on observations obtained at ESO-Paranal (NACO) and HST.
}

LHS $2397 \mathrm{aA}(11: 21: 49,-13: 13: 08)$ is a very low mass star (spectral type M8) at a distance of $14.3 \mathrm{pc}$. This allows us to study it at resolutions of the order of a few AUs $\left(10^{-2}\right.$ arcsec). Optical and NIR photometry was reported by Leggett et al. (2002) and the rotation velocity $\left(v \sin i=20 \mathrm{~km} \mathrm{~s}^{-1}\right)$ and the chromospheric $\left(\mathrm{H}_{\alpha}-E W=29.4 \AA\right)$ activity was studied by Mohanty \& Basri (2003). A BD companion to LHS $2397 \mathrm{aB}$ was discovered by Freed et al. (2003) at a close distance of only $\sim 2.86 \mathrm{AU}$. In this paper we report the astrometric parameters and the photometry as retrieved by observations done with the ESO-VLT 8m telescope "Yepun" and NACO, which consists of the facility adaptive optics system NAOS (Rousset et al. 2002) and the infrared camera CONICA (Lenzen et al. 1998). Our results will be discussed taking into account the previous observations of the same object: images obtained with the adaptive optics system Hokupa'a, at Gemini North (Freed et al. 2003) and images from the HST/WFPC2 archive data (G06345, P.I. Kirkpatrick).

\section{Observations}

Observations of LHS 2397aA and other infrared standard stars were obtained on March 26, 2002. The observations were obtained during the NACO commissioning, and the infrared standard star LHS 2397aA was observed in the $H$ and $K$ s broad band filters with the CONICA S13 camera. LHS 2397aA was 
Table 1. Summary of the astrometric and photometric estimations. In the first three lines the HST/WFPC2 results from the Masciadri et al. (2003) and Freed et al. (2003) analyses are shown. In the following lines the results obtained from AO-Hokupa'a data and the from AO-NACO data are shown.

\begin{tabular}{cccc}
\hline \hline & & This paper & Freed et al. \\
\hline HST/WFPC2 1997/4/12 & Separation (") & $0.255 \pm 0.007$ & $0.270 \pm 0.010$ \\
& Position Angle (degrees) & $90.3 \pm 0.8$ & $82.8 \pm 1.5$ \\
& $\Delta I_{\mathrm{c}}$ & $4.34 \pm 0.14$ & $4.42 \pm 0.17$ \\
\hline AO-HOKUPA 2002/2/7 & Separation (") & - & $0.207 \pm 0.007$ \\
& Position Angle (degrees) & - & $151.9 \pm 1.2$ \\
& $\Delta H$ & - & $3.15 \pm 0.30$ \\
& $\Delta K \mathrm{~s}$ & - & $2.77 \pm 0.10$ \\
\hline AO-NACO 2002/3/26 & Separation (") & $0.164 \pm 0.004$ & - \\
& Position Angle (degrees) & $157.3 \pm 2.0$ & - \\
& $\Delta H$ & $3.03 \pm 0.13$ & - \\
& $\Delta K \mathrm{~s}$ & $2.53 \pm 0.03$ & - \\
\hline
\end{tabular}
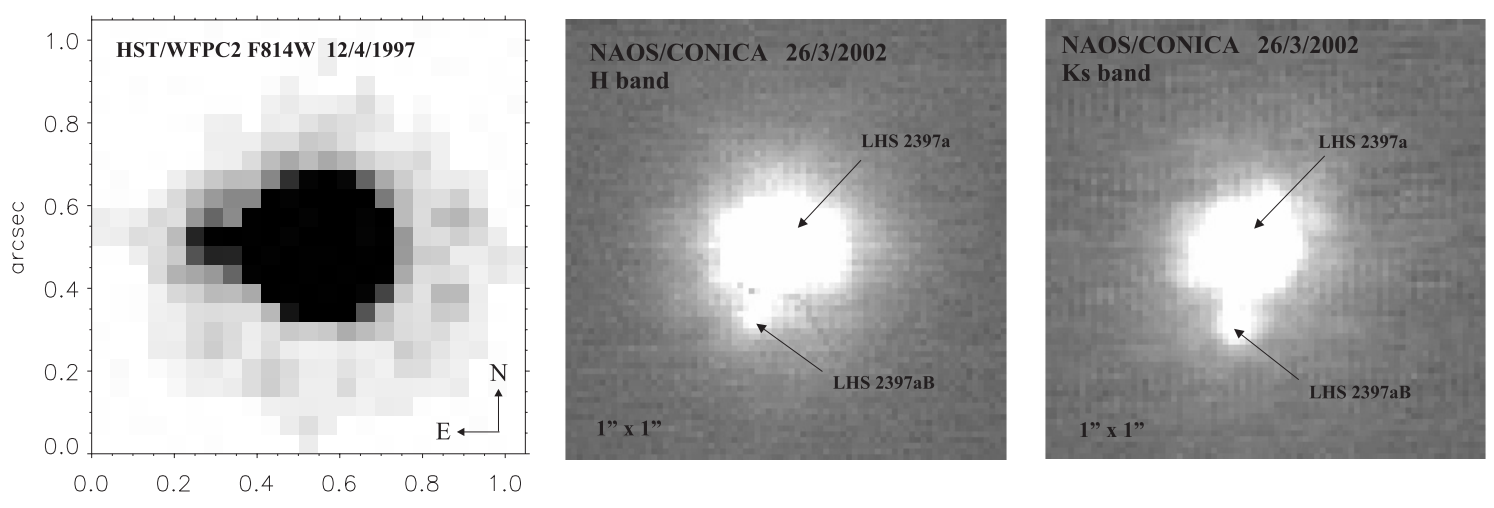

Fig. 1. Left: LHS 2397aA and LHS 2397aB images taken with the HST/WFPC2 camera and the F814W filter on 12/4/1997. Centre and right: LHS $2397 \mathrm{aA}$ and LHS $2397 \mathrm{aB}$ images taken in $H$ (center) and $K$ s (right) broad band with AO-NACO on 26/3/2002. The field of view (FOV) of all the images is $1^{\prime \prime} \times 1^{\prime \prime}$.

observed in a 5-point dither pattern with individual exposure times of $5 \times 2 \mathrm{~s}$ (i.e. $10 \mathrm{~s}$ per dither position) for the $\mathrm{S} 13$ camera (pixel equivalent to 0 ' $^{\prime} 013$ and $14^{\prime \prime} \times 14^{\prime \prime}$ FOV). Observations of other infrared standard stars (FS 18 and FS 20) were obtained in a similar fashion i.e. with the same exposure time and dither position.

A precise knowledge of the pixel scale and and on-sky orientation (instrumental position angle) of CONICA is a prerequisite for a sound determination of the separation and position angle of the LHS 2397aA binary. The lab and on-sky calibration of these parameters is summarized in Brandner (2002). During the commissioning, two independent data sets were obtained to get precise on-sky pixel scales and position angles for CONICA. First, astrometric binaries were observed. For each binary, instrumental positions (pixel coordinates) were compared with true separation and position angle. Secondly, the Galactic Center region was observed multiple times during the commissioning with NACO. Here a global astrometric solution was obtained based on the positions of maser sources in the Galactic Center region (Schödel et al. 2002; Reid et al. 2003). The calibrations obtained with both methods were quite consistent, and yielded for camera S13 the on-sky pixel scale with a precision of $0.22 \%(13.26 \pm 0.03$ mas $)$ and the instrumental position angle with a precision of $\pm 0.5 \mathrm{deg}$.
Our observations were done with a seeing in the range 0 .'68-0.'74, an airmass of $\sim 1.15$ and resulting $F W H M$ equal to $\sim 0$ ' $^{\prime} 078$ in the $K$ band. We selected the PSF stars which were observed under similar conditions (equivalent seeing, comparable $F W H M$ ). Figure 1 shows images of LHS 2397aA taken in the $H$ (centre) and $K$ s (right) broadbands over $1^{\prime \prime} \times 1$ " FOV. The grey table is logarithmic, no low-frequency filtering was done (as in the equivalent images in Freed et al. 2003 - Fig. 1). Figure 1 (left) shows the image taken with the HST/WFPC2 camera in $\mathrm{F} 814 \mathrm{~W}$ that we reduced to complete our analysis.

\section{Data analysis}

A PSF fitting program, conceived to compute the separation, the position angle and the flux ratio of stellar binary systems was used to reduce our data. This program (Bouy et al. 2003) is based on a fit of observed PSF stars trying to reproduce the observed binary system. A detailed description as well as a validation of the technique is described in Bouy et al. (2003). The fitting program is applied to square fields centred on both the scientific targets and the standard stars. The square field measures $64 \times 64$ pixels (corresponding to $0 .^{\prime} 8 \times 00^{\prime} 8$ ). We note that the FS 18 standard is a binary with a separation of 1'.36 i.e. a distance larger than the size of the square field on which the fitting program is applied. This means that the binarity of FS 18 
does not present a problem for our data reduction. We first calculated, in an iterative way, the optimized model (i.e. a synthetic binary) and then we retrieved a rms $\left(\sigma_{\text {res }}\right)$ of the residuals as a quantitative measure on the quality of our fit. The calculation of the astrometric parameters (separation and PA) was done for the $K \mathrm{~s}$ band data in which the binary was well resolved and the SNR was good enough to retrieve reliable values for the astrometric parameters. For the $H$ band data the astrometric parameters derived from the $K$ s band data were fixed and only the brightness ratio was left as a free parameter.

Table 1 shows the astrometric and photometric values retrieved from our AO-NACO data taken in March 2002, by Freed et al. (2003) (February 2002 - AO-Hokupa'a) and by HST/WFPC2 (April 1997). Both the astrometric and photometric NACO values shown in Table 1 are obtained considering the systematic (see further down on the calibration procedure) and statistical errors. The angular separation of $0 \prime^{\prime} 164 \pm 0 .{ }^{\prime} 004$ translates to a physical separation of $2.34 \pm 0.14$ AU.. The absolute magnitude was calculated assuming a distance of $d=$ $14.3 \pm 0.4 \mathrm{pc}$.

The procedure used to calculate the fitting parameters is as follows. For each observation of LHS 2397aA, two sets of "corresponding" PSFs stars (observations of FS 18 and FS 20 with similar $S R$ and $F W H M$ as LHS $2397 \mathrm{aA}$ ) were used to model the observed brightness distribution, and hence to derive the parameters for separation, position angle and flux ratio. The final numbers reported in this paper were computed based on a weighted average of each fitting parameter: mean separation ( $\overline{\mathrm{sep}}$.), position angle $(\overline{\mathrm{pa}})$ and flux ratio $(\bar{q})$. The weights of the statistical estimations are inversely proportional to the rms of the residuals $\left(\sigma_{\text {res }_{i}}\right)$ model-observations found in the fitting process. The index $i$ refers to the library of images and different PSFs.

To calibrate the program we applied it to artificial binaries covering angular separations including the range [0.'1-0.'4] and differences in magnitude between the components in the [0.2-3.2] mag range. We note that the $\Delta m_{K \mathrm{~s}}$ retrieved by our fit before the calibration is equal to $2.17 \mathrm{mag}$ so it belongs to the studied range. A detailed description of the calibration method can be found in Sect. 2.3.2 in Bouy et al. (2003). The calibration procedure permitted us to find the off-sets i.e. a sort of systematic error for each parameter (astrometric and photometric) that we have to add to our statistic estimations.

The results of the calibration procedure gives us an off-set for the angular separation equal to $3.2 \pm 0.003$ mas, the systematic error for the PA is estimated equal to $3.4 \pm 2$ degrees and that for the magnitude difference is $0.36 \pm 0.02 \mathrm{mag}\left(\Delta m_{K \mathrm{~s}}\right)$ and $0.69 \pm 0.06 \mathrm{mag}\left(\Delta m_{H}\right)$. We note that the calibration results are instrument dependent and our estimations are obtained with a not too rich sample (a few PSFs). It is planned to improve the calibration for NACO (an observing program whose task is the search for BDs binaries is in progress with NACO) using a richer sample of PSFs. At the present time we can state that a detailed calibration was been done for HST observations (Bouy et al. 2003) using a rich statistical sample.

Due to the quite high $S R$ of the HST/WFPC2 images, the binary is well resolved and systematic effects in the fitting procedure are small. This means that in this case we do not need
Table 2. Apparent (first 6 lines) and absolute (last 6 lines) magnitude calculated in the $H, K \mathrm{~s}$ and $I_{\mathrm{c}}$ band. The values marked with an asterisk refer to the Freed et al. (2003) paper.

\begin{tabular}{lcc}
\hline \hline & LHS 2397aA & LHS 2397aB \\
\hline$m_{I_{\mathrm{c}}}$ & $14.81 \pm 0.01$ & $19.08 \pm 0.14$ \\
$m_{I_{\mathrm{c}}}^{*}$ & $15.07 \pm 0.03$ & $19.49 \pm 0.17$ \\
$m_{H}$ & $11.32 \pm 0.02$ & $14.35 \pm 0.25$ \\
$m_{H}^{*}$ & $11.32 \pm 0.05$ & $14.47 \pm 0.30$ \\
$m_{K \mathrm{~s}}$ & $10.82 \pm 0.02$ & $13.36 \pm 0.02$ \\
$m_{K \mathrm{~s}}^{*}$ & $10.80 \pm 0.03$ & $13.57 \pm 0.10$ \\
\hline$M_{I_{\mathrm{c}}}$ & $14.03 \pm 0.06$ & $18.30 \pm 0.15$ \\
$M_{I_{\mathrm{c}}}^{*}$ & $14.29 \pm 0.07$ & $18.71 \pm 0.18$ \\
$M_{H}$ & $10.56 \pm 0.14$ & $13.58 \pm 0.18$ \\
$M_{H}^{*}$ & $10.54 \pm 0.08$ & $13.69 \pm 0.31$ \\
$M_{K \mathrm{~s}}$ & $10.05 \pm 0.14$ & $12.58 \pm 0.14$ \\
$M_{K \mathrm{~s}}^{*}$ & $10.03 \pm 0.07$ & $12.80 \pm 0.12$ \\
\hline
\end{tabular}

a calibration for the program (see Bouy et al. 2003). We calculated averaging the values of the four best fits (i.e. the smallest residuals) out of nine fits obtained using as the PSF the following stars: BRI 0021, LHS 2243, TVLM 868 and RG 0050.

Table 2 shows, for the primary (LHS 2397aA) and the companion (LHS 2397aB), the apparent and absolute magnitude in the $H$ and $K$ s bands (NACO and Hokupa'a data) and $I_{\mathrm{c}}$ band (HST/WFPC2 data). The HST handbook states that the F814W filter is a very close approximation to the Johnson-Cousins $I_{\mathrm{c}}$ and colour terms between these filters are very small. We can hence assume that $\mathrm{F} 814 \mathrm{~W}$ magnitudes are a good approximation of the $I_{\mathrm{c}}$ magnitudes. We consider (see Table 1) $\Delta\left(I_{\mathrm{c}}\right)=$ $\Delta F 814 W+0.06 \mathrm{mag}$ as in the Freed paper.

\section{Discussion}

The photometric results retrieved in our analysis are in good agreement with Freed et al. (2003). The few discrepancies are not larger than the error bar with which the $\Delta(m)$ is estimated for the ground-based observations. Our estimation of $\Delta\left(m_{I_{\mathrm{c}}}\right)$ is equal to $4.34 \pm 0.14 \mathrm{mag}$ in good agreement with that estimated by Freed et al. (4.42 $\pm 0.17 \mathrm{mag})$.

The parameter ORIENTAT in the fits file header of the HST/WFPC2 image gives the position of the north with respect to the $\mathrm{y}$ axis. Using this offset angle, and the measured instrumental $\mathrm{PA}_{\text {inst }}=320^{\circ} .3$, we get $\mathrm{PA}=$ ORIENTAT $\left(360^{\circ}-\mathrm{PA}_{\text {inst }}\right)=90^{\circ} 3$. We note that this differs from the $82^{\circ} .8$ reported by Freed et al. (2003). Since the binary is well resolved, the discrepancy is most likely due to a problem in the transformation from instrumental to true PA as done by Freed et al.

We note that the angular separation measured in the different periods seems to decrease with the time. This could indicate an inclined elliptical orbit of the companion. Nevertheless, the epoch difference between our observations (AO-NACO) and the one by Freed et al. (AO-Hokupa'a) is just one month. This seems to be a quite small temporal difference to justify a dif-

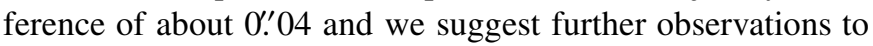
confirm the trend or to get a better estimate of the angular separation. Besides this we note that, because of the systematic 
error, the uncertainty on the position angle is about 2 degrees. We think that it is premature to try to retrieve an orbit trajectory. Under the hypothesis of a circular orbit the companion should complete a revolution in about $26.5 \mathrm{yr}$ (13.6 deg/yr). More reliable orbital parameters will become available with further observations to be made in the incomin years.

\section{Conclusions}

The most important conclusion of our study is that the analysis of observations of LHS 2397aA taken in March 2002 with AO-NACO gives astrometric and photometric results in good agreement with the previous ones obtained with another ground-based AO-system (Hokupa'a) although the reduction of data was made in two different ways. The difference in the photometry is smaller than the uncertainty of the measurements. In conclusion we can confirm the results of Freed et al. (2003) for photometry, age and spectral type. We suggest further observations to confirm the angular separation of 0.164 estimated in our analysis and to attemp a calculation of the companion orbit. We note that the PA derived by us for the HST data differs from the PA quoted by Freed et al. Our determination suggests a longer orbital period.

Acknowledgements. These data are part of the NACO commissioning data. We acknowledge the staff who took part in the observations. We acknowledge the anonymous referee for the useful commentaries and Dave Butler for the improvements suggested in the English form.

\section{References}

Bouy, H., Brandner, W., Martin, E. L., et al. 2003, AJ, 126, 1526

Brandner, W. 2000, NACO Commissioning Report - Comm 4 VLT-TRE-ESO-14200-2817

Burgasser, A. J., Kirkpatrick, J. D., Reid, I. N., Brown, M. E., \& Miskey, C. L. 2003, ApJ, 568, 512

Close, L., Siegler, N., Freed, M., \& Biller, B. 2003, ApJ, 587, 407

Elmegreen, B. G. 1999, ApJ, 522, 915

Freed, M., Close, L. M., \& Siegler, N. 2003, ApJ, 584, 453

Gizis, J., Kirkpatrick, J. D., Burgasser, A., et al. 2001, ApJ, 551, L163

Legget, S. K., Golimowski, D. A., Fan, X., et al. 2002, ApJ, 564, 452

Lenzen, R., Hofmann, R., Bizenberger, P., \& Tusche, A. 1998, SPIE, 3354, 606

Kirkpatrick, J. D., Dahan, C. C., Monet, D. G., et al. 2001, AJ, 121, 3235

Koerner, D. W., Kirkpatrick, J. D., McElwain, M. W., \& Bonaventura, N. R. 1999, ApJ, 526, L25

Marcy, G., Butler, R. P., Fischer, D. A., \& Vogt, S. S. 2003, ASP Conf. Ser. Scientific Frontiers in Research on Extrasolar Planets, ed. D. Deming, \& S. Seager, in press

Mohanty, S., \& Basri, G. 2003, ApJ, 583, 451

Pickett, B. K., Durisen, R. H., Cassen, P., \& Mejia, A. C. 2000, ApJ, 540, L95

Potter, D., Martin, E. L., Cushing, M. C., et al. 2002, ApJ, 567, 133

Reid, M. J., Menten, K. M., Genzel, R., et al. 2003, ApJ, 587, 208

Reipurth, B., \& Clarke, C. 2001, AJ, 122, 432

Rousset, G., Lacombe, F., Puget, P., et al. 2000, SPIE, 4007, 72 